\title{
The effect of stimulus intensity on neural envelope tracking
}

Eline Verschueren ${ }^{a, *}$, Jonas Vanthornhout ${ }^{a}$ and Tom Francart ${ }^{a}$

${ }^{a}$ Research Group Experimental Oto-rhino-laryngology (ExpORL), Department of Neurosciences, KU Leuven - University of Leuven, 3000 Leuven, Belgium

Correspondence*:

Eline Verschueren

Herestraat 49, bus 721,3000 Leuven, Belgium

eline.verschueren@kuleuven.be

Declarations of interest: none 


\section{ABSTRACT}

Objectives In recent years, there has been significant interest in recovering the temporal envelope of a speech signal from the neural response to investigate neural speech processing. The research focus is now broadening from neural speech processing in normal-hearing listeners towards hearing-impaired listeners. When testing hearing-impaired listeners, speech has to be amplified to resemble the effect of a hearing aid and compensate for peripheral hearing loss. Today it is not known with certainty how or if neural speech tracking is influenced by sound amplification. As these higher intensities could influence the outcome, we investigated the influence of stimulus intensity on neural speech tracking.

Design We recorded the electroencephalogram (EEG) of 20 normal-hearing participants while they listened to a narrated story. The story was presented at intensities from 10 to $80 \mathrm{~dB}$ A. To investigate the brain responses, we analyzed neural tracking of the speech envelope by reconstructing the envelope from the EEG using a linear decoder and by correlating the reconstructed with the actual envelope. We investigated the delta $(0.5-4 \mathrm{~Hz})$ and the theta $(4-8$ $\mathrm{Hz}$ ) band for each intensity. We also investigated the latencies and amplitudes of the responses in more detail using temporal response functions, which are the estimated linear response functions between the stimulus envelope and the EEG.

Results Neural envelope tracking is dependent on stimulus intensity in both the TRF and envelope reconstruction analysis. However, provided that the decoder is applied to the same stimulus intensity as it was trained on, envelope reconstruction is robust to stimulus intensity. Besides, neural envelope tracking in the delta (but not theta) band seems to relate to speech intelligibility. Similar to the linear decoder analysis, TRF amplitudes and latencies are dependent on stimulus intensity: The amplitude of peak $1(30-50 \mathrm{~ms})$ increases, and the latency of peak 2 (140-160 ms) decreases with increasing stimulus intensity.

Conclusion Although brain responses are influenced by stimulus intensity, neural envelope tracking is robust to stimulus intensity when using the same intensity to test and train the decoder. Therefore we can assume that intensity will not be a confounder when testing hearing-impaired participants with amplified speech using the linear decoder approach. In addition, neural envelope tracking in the delta band appears to be correlated with speech intelligibility, showing the potential of neural envelope tracking as an objective measure of speech intelligibility.

Keywords: neural coding, natural speech, stimulus intensity, EEG, speech understanding 


\section{INTRODUCTION}

In recent years, there has been significant interest in recovering the temporal envelope of a speech signal from the neural response to investigate neural speech processing (e.g., Ding and Simon, 2012b; O'Sullivan et al., 2015; Das et al., 2016). The temporal envelope contains syllable, word, and sentence boundaries and is considered to be essential for speech understanding (Shannon et al., 1995). A growing body of research shows that the quality of reconstruction of the temporal envelope from the neural responses, so-called neural envelope tracking, is related to speech intelligibility (e.g. Ding and Simon, 2013; Di Liberto et al. 2018; Vanthornhout et al., 2018; Lesenfants et al., 2019; Decruy et al., 2019; Iotzov and Parra, 2019; Verschueren et al., 2020): The better the acoustic speech envelope is encoded in the brain, the better the speech is understood.

The research focus is now broadening from neural speech processing in normal-hearing listeners towards hearing-impaired listeners. Several studies already compared normal-hearing to hearing-impaired listeners: Petersen et al. (2017) and Presacco et al. (2019) reported no significant difference in neural envelope tracking of the attended speech between normal-hearing and hearing-impaired listeners. Similar, Vanheusden et al. (2020) did not find a significant difference between cortical tracking of speech presented with and without hearing aids at an intelligible level in participants with mild to moderate hearing loss. Decruy et al. (2020b), on the other hand, showed increased neural envelope tracking in hearing-impaired listeners compared to normal-hearing peers. The results of Mirkovic et al. (2019) are in line with Decruy et al. (2020b) and suggest (no statistics were reported) higher envelope tracking for the attended speech in participants with hearing loss. A critical remark is that in three (Petersen et al., 2017; Mirkovic et al., 2019; Decruy et al., 2020b) out of four studies speech was (linearly) amplified. Amplification was applied to the stimuli to resemble the effect of a hearing aid and compensate peripheral hearing loss. It is not known with certainty how or if neural envelope tracking is influenced by amplification. Therefore it is difficult to disentangle whether the reported results above are an effect of hearing loss or sound amplification. Besides, Verschueren et al. (2019) reported increased neural envelope tracking in the delta band with increasing speech understanding by varying the intensity of the presented speech in cochlear implant (CI) users. Again, it is difficult to distinguish whether neural envelope tracking decreases because of decreasing speech intelligibility or stimulus intensity.

In this study, we investigate the influence of stimulus intensity on neural envelope tracking in normalhearing listeners. We hypothesize that neural envelope tracking is sensitive to the absolute level at which the speech is presented. The link between stimulus intensity and cortical auditory evoked potentials (CAEP) has been thoroughly investigated and established using non-speech stimuli. In short, the amplitudes of the CAEP peaks increase, and the latencies decrease with increasing stimulus intensity (e.g., Moore and Rose, 1969; Picton et al., 1970; Adler and Adler, 1989, Billings et al., 2007). In addition, a similar effect of stimulus intensity on brainstem responses has been shown using transient stimuli. With decreasing stimulus intensity, the latencies of the auditory brainstem response (ABR) peaks increase, and the amplitudes of the response peaks decrease until the hearing threshold is reached and no response is present anymore (Hall. 2007). In line with this, Picton et al. (2003) and Van Eeckhoutte et al. (2016) reported a similar decreasing amplitude pattern with decreasing stimulus intensity using auditory steady-state responses (ASSR).

In summary, all these studies show an effect of stimulus intensity on auditory evoked potentials in normal-hearing listeners. However, none of them used running speech as a stimulus. To our knowledge, only one study explored the effect of variations in stimulus intensity on neural speech tracking using running speech: Ding and Simon (2012a) presented speech in the presence of a competing talker. They fixed the intensity level of one speech stream, either the attended or the background speaker while reducing the other speech stream up to $8 \mathrm{~dB}$. They concluded that neural tracking of a speech stream was not influenced by the intensity change of that stream or the other stream. Based on these promising results using a competing talker paradigm, we investigated the effect of the absolute level of stimulus intensity on neural envelope tracking for a wide range of intensity variations. This could shed some light on the effects reported in hearing-impaired listeners: Did Decruy et al. (2020b) and Mirkovic et al. (2019) find enhanced neural envelope tracking in hearing-impaired participants listening to amplified sound because of increased 
stimulus intensity or because of a different factor? Was the increase of neural envelope tracking with increasing stimulus intensity shown by Verschueren et al. (2019) in CI users driven by stimulus intensity or speech understanding?

\section{MATERIAL AND METHODS}

\subsection{Participants}

Twenty participants aged between 19 and 24 years ( 4 men and 16 women) took part in the experiment after providing informed consent. Participants had Dutch as their mother tongue and were all normal-hearing, confirmed with pure tone audiometry (thresholds $\leq 25 \mathrm{~dB} \mathrm{HL}$ at all octave frequencies from $125 \mathrm{~Hz}$ to $8 \mathrm{kHz}$ ). The study was approved by the Medical Ethics Committee UZ Leuven / Research (KU Leuven) with reference S57102.

\subsection{EEG experiment}

Recordings were made in a soundproof and electromagnetically shielded room. Speech was presented bilaterally using APEX 4.0 (Francart et al., 2008), an RME Multiface II sound card (Haimhausen, Germany) and Etymotic ER-3A insert phones (Illinois, USA). The setup was calibrated using a $2 \mathrm{~cm}^{3}$ coupler of the artificial ear (Brüel \& Kjær 4152, Denmark). EEG was recorded with a 64-channel BioSemi ActiveTwo EEG recording system was at a sample rate of $8192 \mathrm{~Hz}$. Participants sat in a comfortable chair and were asked to move as little as possible during the recordings.

The story used during the EEG measurement was 'The Casual Vacancy' by J.K. Rowling, narrated in Dutch by Nelleke Noordervliet. This story was used to investigate the effect of stimulus intensity (this study) and speech rate (not discussed in this paper) on neural speech tracking. The total measurement time for the intensity part (this study) was 46 out of 96 minutes. We inserted a break between the two parts and several smaller breaks within a part if needed. Ten participants started the EEG experiment by listening to the stimulus intensity part (this study). The remaining 10 participants started with the speech rate part.

For this study, the story was split into 6 longer blocks of $\approx 5$ minutes [mean $=316$ seconds, sd $=$ 2.8 seconds] and 8 shorter blocks of $\approx 2$ minutes [mean $=124$ seconds, sd $=2.6$ seconds] which were presented in chronological order. The 5-minute blocks were presented randomly at 30,60, and $70 \mathrm{~dB} \mathrm{~A}$ in the beginning and at the end of each session. This way, every intensity was applied twice (once in the beginning and once at the end) to obtain 10 minutes of speech at the same intensity, which was necessary for further analysis. The short 2-minute blocks were presented at 8 different intensities randomly selected from a fixed list, containing 10, 20, 30, 40, 50, 60, 70, $80 \mathrm{~dB}$ A. An overview of the different conditions is shown in Figure 1. We presented the 5-minute blocks on purpose in the beginning and at the end to ensure the decoders we would create based on this data were optimized to decode the different 2-minute speech segments in between. We know, for example, attention and listening effort could shift during the measurement, which could influence neural speech tracking (Ding and Simon, 2012a; Vanthornhout et al., 2019; Lesenfants and Francart, 2020). To ensure understanding of the storyline, the participants read the story summary before the experiment started. Also, we asked content questions after each 2- or 5-minute block to maximize the participants' attention and motivation. In addition, speech intelligibility was measured after each block using a rating method where the participants were asked to rate their speech 
understanding on a scale from 0 to $100 \%$ following the question 'Which percentage of the story did you understand?'. Two out of 20 participants, participating in the pilot study, did not listen to speech intensities higher than $60 \mathrm{~dB} \mathrm{~A}$. Based on the pilot study results, we decided to enlarge the range of stimulus intensities with 70 and $80 \mathrm{~dB}$ A for further measurements.

\section{Overview EEG session}

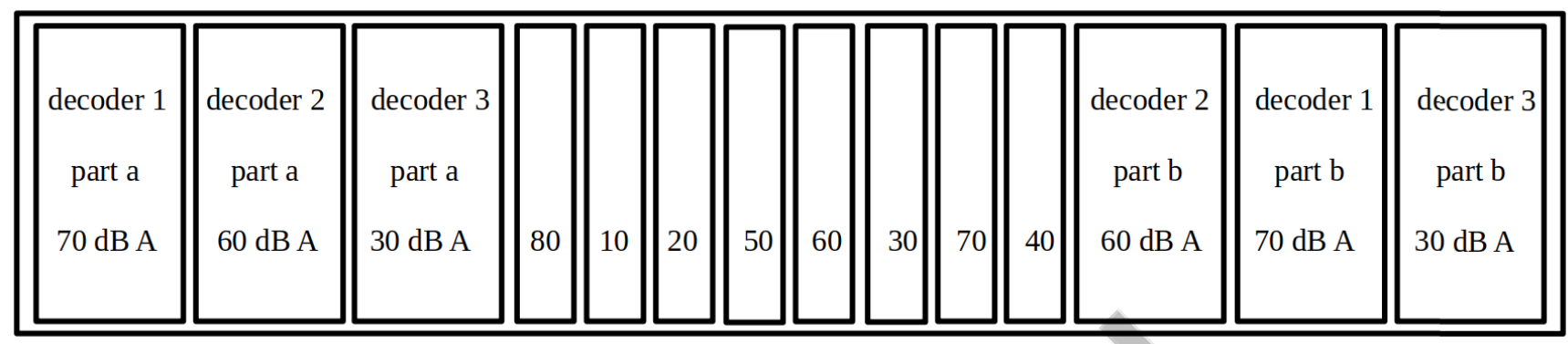

Figure 1. Overview of an example test session. The numbers in each block indicate the presented stimulus intensity in $\mathrm{dB}$ A. Six longer 5-minute blocks were presented at 30, 60, and $70 \mathrm{~dB}$ A. Every intensity was used twice (part a and b) to obtain 10 minutes of speech at a same intensity. Eight shorter blocks were presented at intensities varying from 10 to $80 \mathrm{~dB}$ A. Each participant thus listened to 46 minutes of speech during the EEG session.

\subsection{Signal processing}

In this study, we measured neural envelope tracking and related this to stimulus intensity. Neural envelope tracking was calculated using an envelope reconstruction approach 2.3.1) and temporal response functions (2.3.2.

\subsubsection{Envelope reconstruction}

Details of the signal processing steps applied to the EEG data to measure neural envelope tracking using envelope reconstruction are described carefully in Vanthornhout et al. (2018) and Verschueren et al. (2020)).

In short, we extracted the speech envelope from the original signal according to Biesmans et al. (2017) using a gamma-tone filter bank followed by a power law, and band-pass filtered the envelope in the delta (0.5-4 Hz) and theta $(4-8 \mathrm{~Hz})$ frequency band. Second, we reconstructed the EEG speech envelope with a linear decoder estimated using ridge regression and the mTRF toolbox similar to Lalor et al. (2006), Crosse et al. (2016) and Vanthornhout et al. (2018), taking all 64 EEG channels and their time-lagged variants from 0 to $250 \mathrm{~ms}$ into account. In summary, the following steps were applied: The EEG data was downsampled from $8192 \mathrm{~Hz}$ to $256 \mathrm{~Hz}$ using the Matlab resample function with an anti-aliasing filter. Next, artifact rejection was applied using the multi-channel Wiener filter (Somers et al., 2018), the signal was band-pass filtered similar to the acoustic speech envelope and the sample rate was further decreased from $256 \mathrm{~Hz}$ to $128 \mathrm{~Hz}$. Only the longer 10-minute blocks at 30, 60, and $70 \mathrm{~dB}$ A were used to train the decoder. The shorter 2-minute blocks with varying intensity from 10 to $80 \mathrm{~dB}$ A were used to apply the decoder. Consequently, the decoder could be applied on data of the same or a different intensity as it was trained on. 
We applied leave-one-out cross-validation, after normalization, to investigate training and testing on the same stimulus intensity on the longer 10-minute speech blocks (Figure 2, upper panel). We always used 1 minute for testing and the remaining 9 minutes for training, resulting in 30 ( 3 stimulus intensities x 10 cross-validations) reconstructed envelopes per participant. To investigate training and testing on a different intensity, we trained 2 decoders on the entire 10-minute blocks at 30 and $60 \mathrm{~dB}$ A. These decoders were used to reconstruct the envelope in the shorter 2-minute story blocks, after normalization, resulting in 16 (2 decoders x 8 stimulus intensities) envelopes per participant (Figure 2, lower panel).

Finally, to quantify neural envelope tracking, we correlated the speech envelope obtained from this reconstruction analysis with the actual speech envelope using bootstrapped Spearman correlations. Only the median of these bootstrapped correlations per condition and participant is used for further analysis. Besides, the significance level of the correlation was calculated by correlating random permutations of the real and reconstructed envelope 1000 times and taking percentile 2.5 and 97.5 to obtain a $95 \%$ confidence interval.

Same intensity in decoder and test stimulus

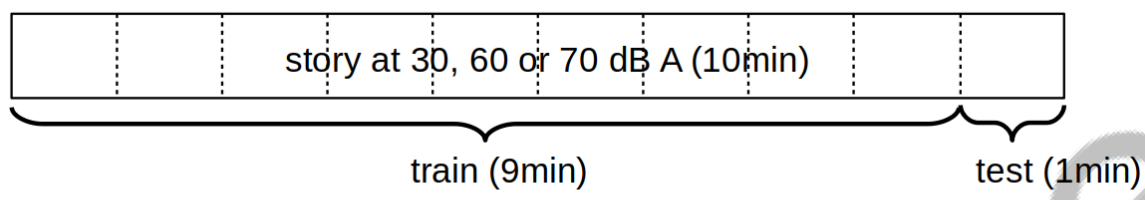

Different intensity in decoder and test stimulus

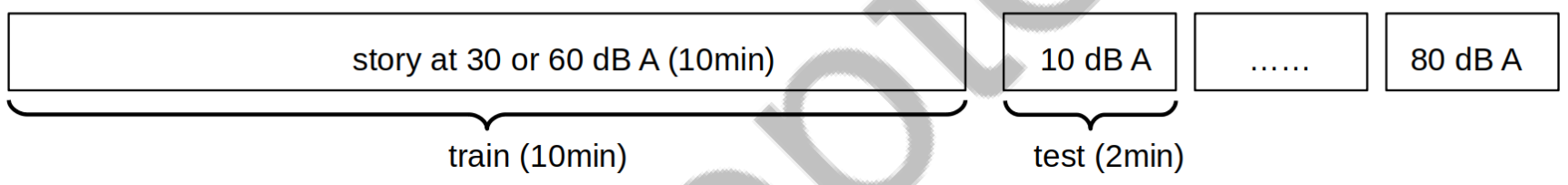

Figure 2. Overview of the 2 analysis methods used to reconstruct the envelope from the EEG response. The upper panel shows the leave-one-out cross-validation on the longer 10-minute speech blocks where decoder and test stimulus have the same intensity. The lower panel shows the decoder approach where a decoder is trained on a different stimulus (10-minute block) and applied on the 2-minute blocks.

\subsubsection{Temporal response function estimation}

The main advantage of the envelope reconstruction analysis is integrating all EEG channels and timelagged variants, leading to a robust prediction. This integration advantage is at the same time also a disadvantage: no interpretation is possible per electrode channel and latency (Haufe et al., 2014). Therefore temporal response function (TRF) estimations are a valuable additional analysis giving some insights at a channel- and latency-level. In addition, no model assumptions are made as with decoders. A TRF is a simplified brain model that describes how the brain, if it were a linear filter, would process the acoustic speech envelope of the stimulus to obtain the recorded EEG signal.

We calculated a TRF for every electrode channel in every participant at 30,60 , and $70 \mathrm{~dB} \mathrm{~A}$. We only included the longer 10-minute blocks because of the amount of data needed to obtain a good prediction. The first signal processing steps are identical to the envelope reconstruction model. Only the filtering was done within a broader band $(0.5-25 \mathrm{~Hz})$. Next, TRFs were calculated using the boosting algorithm using the Eelbrain source code (Brodbeck, 2017) as described in detail by David et al. (2007). In summary, boosting is an iterative algorithm using the mean-squared error (MSE) to find the most optimal prediction for the 
given data. After calculation, the TRFs were convolved with a Gaussian kernel of 5 samples long (SD=2) to smooth over time lags.

\subsection{Statistical Analysis}

Statistical analysis was performed using MATLAB (version R2018a) and R (version 3.4.4) software. The significance level was set at $\alpha=0.05$ unless otherwise stated.

To investigate the effect of stimulus intensity on neural envelope tracking we created a linear mixed effect model (LME) per filter band with the following general formula:

$$
\text { EnvTrack } \sim \text { TestInt }+ \text { TrainInt }+ \text { TestInt }: \text { TrainInt }+ \text { random }=\text { participant }
$$

where "EnvTrack" refers to the Spearman correlation between the reconstructed and the acoustic envelope and "TestInt" refers to the intensity the test stimulus was presented at. We added a fixed and interaction effect of "TrainInt", which is the intensity on which the decoder was trained. In addition, a random effect of intercept of the participants was included in the model to allow neural tracking to be higher or lower per participant. To control if every chosen fixed and random effect benefited the model, the Akaike Information Criterion (AIC) was calculated. Unstandardized regression coefficients (beta) with $95 \%$ confidence intervals and $\mathrm{p}$-value are reported in the results section.

Furthermore, we compared dependent samples using a non-parametric Wilcoxon signed-rank test. To compare multiple dependent samples with incomplete observations, we used the Skillings-Mack statistic (Skillings et al., 1981), an extension of Friedman's test (e.g., neural envelope tracking at 30, 60 and 70 dB A: not all participants listened to $70 \mathrm{~dB}$ A speech as mentioned in section 2.2).

\section{RESULTS}

We analyzed behavioral speech intelligibility as a function of stimulus intensity (section 3.1). Next, we investigated the neural responses to the speech signal at different stimulus intensities in two ways. First, we reconstructed the EEG envelope using a decoder and correlated this reconstructed envelope with the actual acoustic envelope (section 3.2). Second, we calculated TRFs to investigate the neural response per channel over time (section 3.3).

\subsection{Behavioral speech intelligibility}

To investigate the effect of stimulus intensity on speech intelligibility, we asked the participants to rate their speech intelligibility after each presented 2- and 5-minute block of the story. Figure 3 shows that speech intelligibility increases with increasing stimulus intensity until reaching a plateau at $\approx 30$ $\mathrm{dB}$ A. Fitting a sigmoid function on the data shows that participants understand $50 \%$ of the presented speech signal at $17.79 \mathrm{~dB}$ A (midpoint of the sigmoid function). Inter-subject variability depends on the intensity conditions (Levene's test, $\mathrm{F}=7.29, \mathrm{p}<0.001$ ) with the largest variability at an intensity of $20 \mathrm{~dB} A$ $(\mathrm{sd}=22.49)$ when comparing ratings for the 2 -minute blocks (the 5-minute block ratings were excluded from the analysis because they were used to train the decoder). 


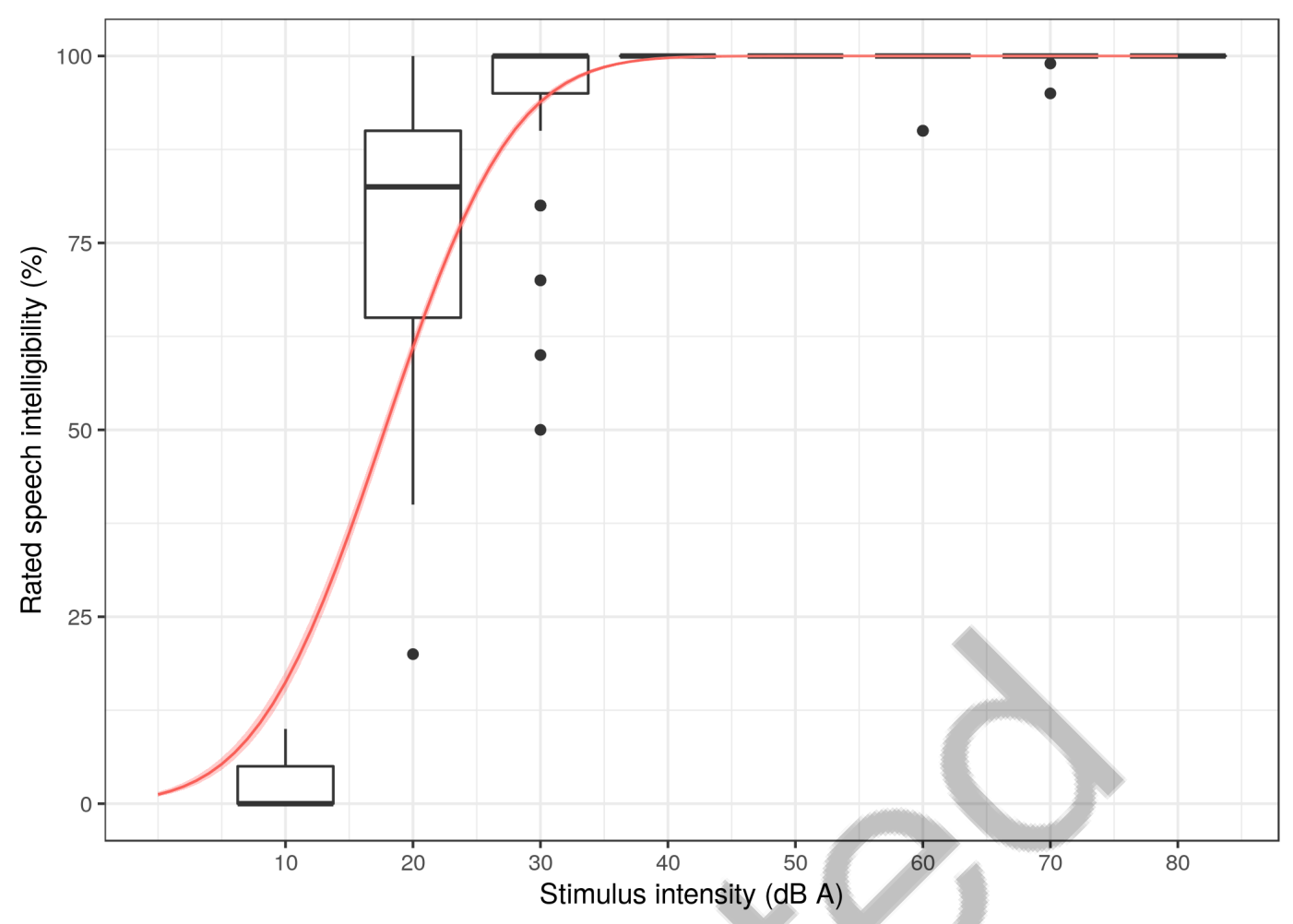

Figure 3. Rated speech intelligibility as a function of stimulus intensity. Rated speech intelligibility increases with increasing stimulus intensity, saturating at $30 \mathrm{~dB}$ A. Variability between participants is largest in the $20 \mathrm{~dB}$ A condition. The red line shows the sigmoid fitted to the data \pm the error of the fit of the model.

\subsection{Effect of stimulus intensity on envelope reconstruction}

\subsubsection{Training and testing on the same stimulus intensity}

Figure 4 shows the the leave-one-out cross-validation results on the longer 10-minute speech blocks. The train and test stimulus were presented at the same stimulus intensity. No significant difference was found in neural envelope tracking between a stimulus presented at 30,60 , or $70 \mathrm{~dB} A$ in the delta $(\mathrm{p}=0.55$, Skillings-Mack statistic $=1.20)$ or theta band $(p=0.52$, Skillings-Mack statistic $=1.30)$. 

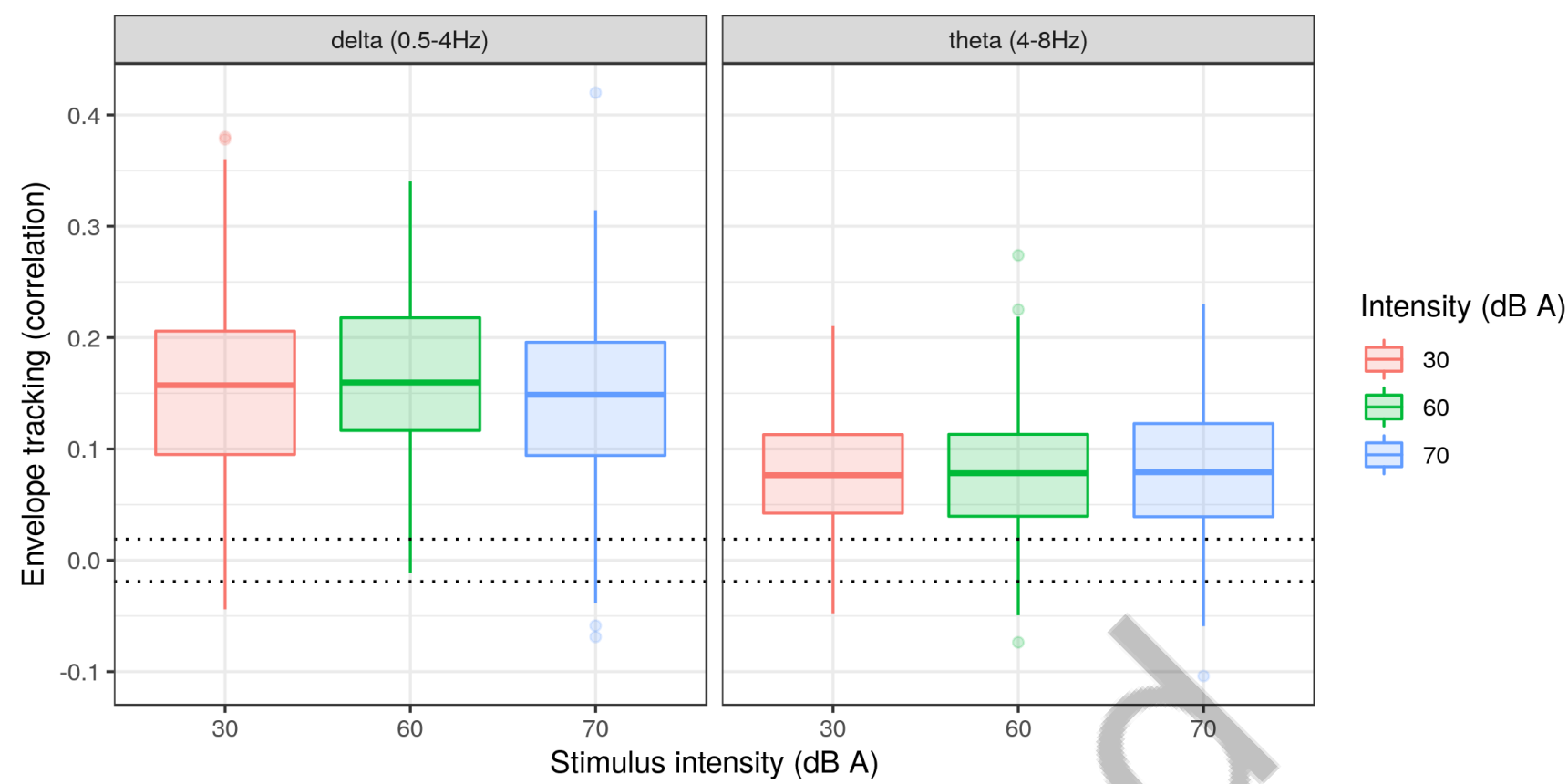

Figure 4. Envelope tracking as a function of stimulus intensity when using the same intensity to train and test the decoder. No significant difference in envelope tracking was found between a stimulus presented at 30,60 , and $70 \mathrm{~dB}$ A. The dotted lines show the significance level of the correlation.

\subsubsection{Training and testing on a different stimulus intensity}

For the next analysis, we trained a decoder for the delta and theta band at 30 and $60 \mathrm{~dB} \mathrm{~A}$, using the story's longer 10-minute blocks. These decoders were applied on the shorter 2-minute blocks with varying intensities from 10 to $80 \mathrm{~dB}$ A, resulting in 32 envelopes ( 2 decoders x 2 filter bands x 8 stimulus intensities) per participant.

Figure 5 shows an increase of neural envelope tracking with increasing stimulus intensity in the delta band $(\mathrm{p}<0.001, \mathrm{~b}=0.0005, \mathrm{CI}(95 \%): \pm 0.0002, \mathrm{LME})$. However, when excluding the $10 \mathrm{~dB} \mathrm{~A}$ (median speech intelligibility $=2.6 \%$ ) condition from statistical analysis and keeping all other remaining conditions with a speech intelligibility level of $50 \%$ or more, no significant effect of stimulus intensity on neural envelope tracking can be found $(\mathrm{p}=0.2, \mathrm{~b}=-0.0002, \mathrm{CI}(95 \%)$ : $\pm 0.0003, \mathrm{LME})$. The stimulus intensity on which the decoder was trained does not add extra information to the model in the delta band (LME, AIC $=-874.06$ versus $\mathrm{AIC}=-874.63$ ).

In the theta band, in contrast to the delta band, the effect of stimulus intensity on neural envelope tracking $(\mathrm{p}<0.001, \mathrm{~b}=0.0006, \mathrm{CI}(95 \%): \pm 0.0002, \mathrm{LME})$ does not disappear when removing the $10 \mathrm{~dB}$ A condition $(\mathrm{p}<0.001, \mathrm{~b}=-0.0008, \mathrm{CI}(95 \%): \pm 0.0004, \mathrm{LME})$. Moreover, the stimulus intensity on which the decoder was trained influences the absolute level of neural envelope tracking $(\mathrm{p}<0.001, \mathrm{~b}=-0.0732, \mathrm{CI}(95 \%)$ : $\pm 0.0273, \mathrm{LME})$ and the slope of neural envelope tracking over stimulus intensity $(\mathrm{p}<0.001, \mathrm{~b}=0.0017$, $\mathrm{CI}(95 \%): \pm 0.0005, \mathrm{LME})$. Figure 5 shows that, in the theta band, neural envelope tracking increases with increasing stimulus intensity for the $60 \mathrm{~dB}$ A decoder (slope LME $=0.96 \times 10^{-3}$ ). On the other hand, for the $30 \mathrm{~dB}$ A decoder, neural envelope tracking decreases with increasing stimulus intensity (slope LME = $-0.75 \times 10^{-3}$ ). In more detail, a Wilcoxon signed-rank test reveals that the envelope of $60 \mathrm{~dB}$ A speech can be reconstructed with higher accuracy using a decoder trained at $60 \mathrm{~dB}$ A compared to a decoder trained at 
$30 \mathrm{~dB}$ A $(\mathrm{p}=0.008, \mathrm{CI}(95 \%)=[-0.072 ;-0.027], \mathrm{n}=20$, Wilcoxon signed-rank test $)$. Similarly, the envelope of $30 \mathrm{~dB}$ A speech can be reconstructed with higher accuracy using a decoder trained at $30 \mathrm{~dB}$ A compared to a decoder trained at $60 \mathrm{~dB} \mathrm{~A}(\mathrm{p}<0.001, \mathrm{CI}(95 \%)=[0.012 ; 0.055], \mathrm{n}=20$, Wilcoxon signed-rank test $)$. In summary, a decoder trained on a low intensity improves envelope reconstruction at low intensities, while a decoder trained on a high intensity improves envelope reconstruction at high intensities.

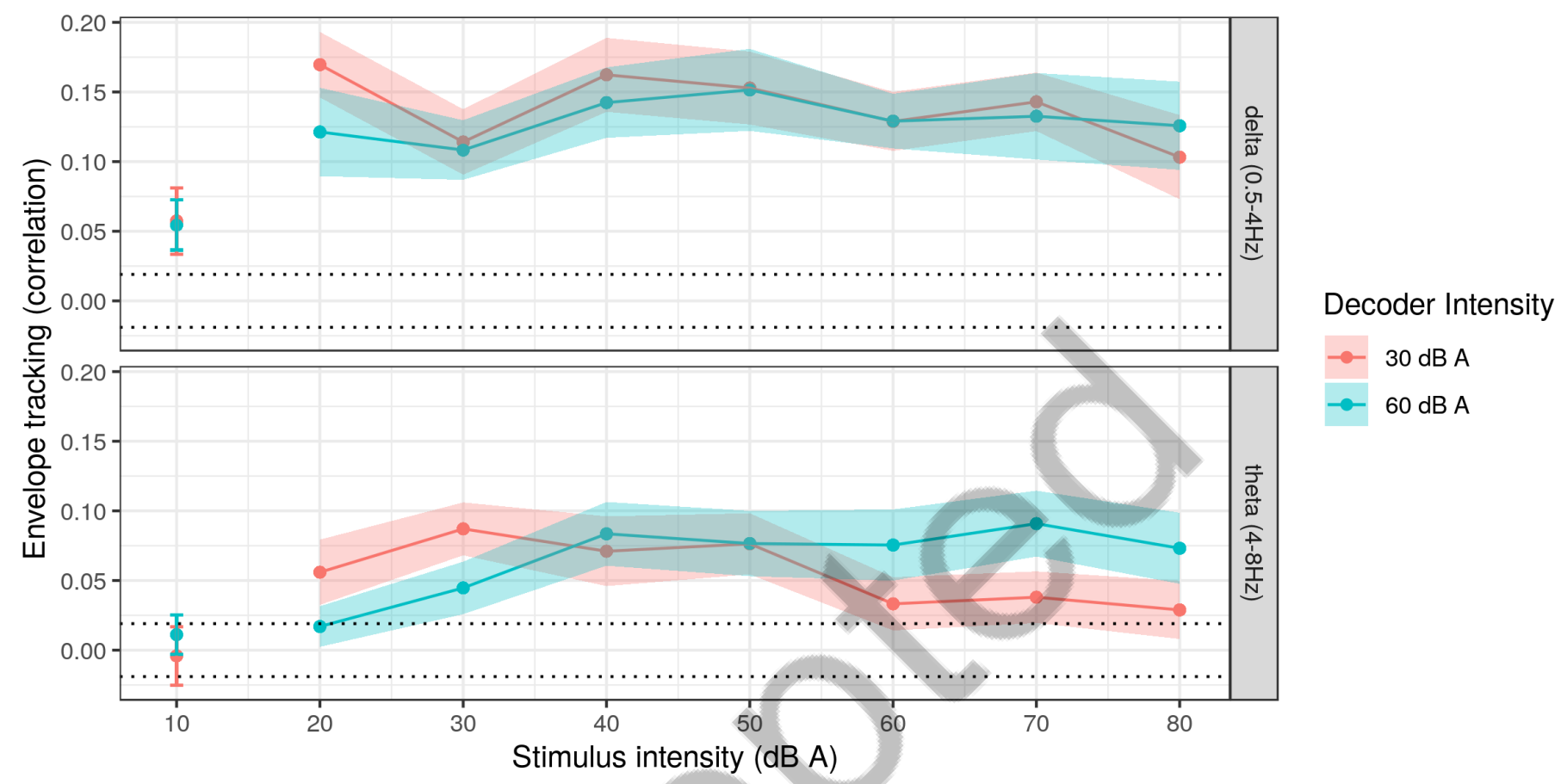

Figure 5. Envelope tracking as a function of stimulus intensity when using a different intensity to train and test the decoder. Envelope tracking is not influenced by stimulus intensity in the delta band. The theta band tracking increases with increasing stimulus intensity for a $60 \mathrm{~dB}$ A decoder but decreases for a 30 $\mathrm{dB}$ A decoder. The solid line shows the median over subjects. The shading is 2 times the standard error. The dotted lines show the significance level of the correlation.

\subsubsection{Effect of speech intelligibility on envelope reconstruction}

Besides comparing stimulus intensity to neural envelope tracking, it is also interesting to analyze the potential relation between actual speech intelligibility and neural envelope tracking. To investigate this, we selected the condition with the most inter-subject variability: intensity $=20 \mathrm{~dB} A$ (analysis in section 3.1). Within this condition, speech intelligibility varied from 20 to $100 \%$ between participants while stimulus intensity remained constant. Based on the previous results showing that best envelope reconstructions are obtained when using a decoder trained on the same or a similar intensity, we selected the $30 \mathrm{~dB} A$ decoder to reconstruct the envelope at $20 \mathrm{~dB}$ A (There was not sufficient data to train a decoder at $20 \mathrm{~dB} \mathrm{~A}$ ). Figure 6 shows that neural envelope tracking increases with increasing speech intelligibility in the delta band $(\mathrm{r}=0.59, \mathrm{p}=0.007$, Spearman rank correlation): The better a participant can understand very soft speech, the higher neural envelope tracking for that participant. In the theta band, no significant effect was found $(\mathrm{r}=0.02, \mathrm{p}=0.94$, Spearman rank correlation $)$. 


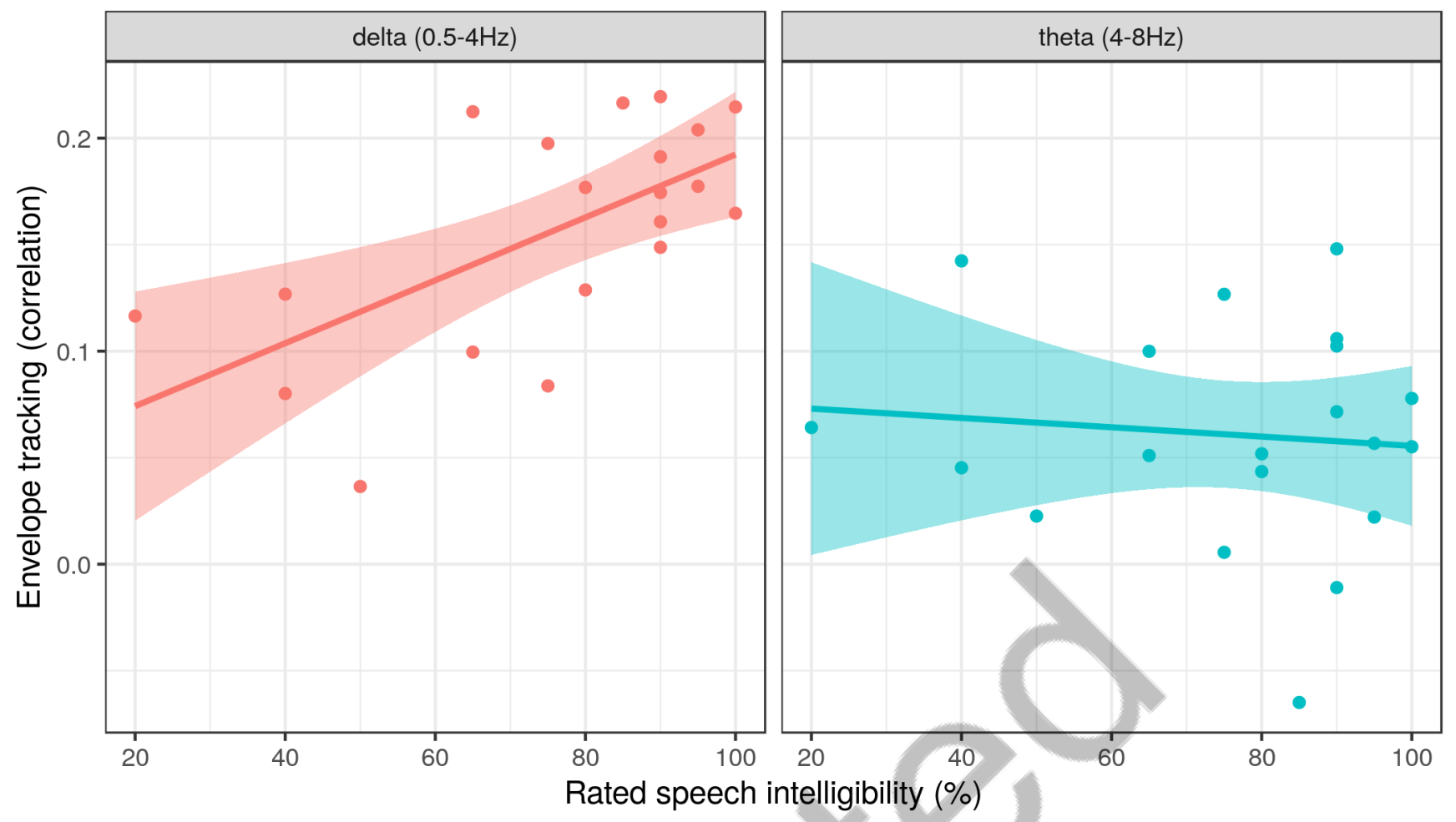

Figure 6. Envelope tracking increases with increasing speech intelligibility at a stimulus intensity of 20 $\mathrm{dB} A$ in the delta band, but not in the theta band. Every dot represents a participant. The solid line shows the linear model fit per filter band. The shading represents the $95 \%$ confidence level interval for the predictions of the linear model.

\subsection{Effect of stimulus intensity on TRFs}

The envelope reconstruction analysis showed that neural envelope tracking is not significantly influenced by stimulus intensity when using the same intensity to train and test the decoder. However, when training and testing on a different stimulus intensity, the quality of envelope reconstruction in the theta band depends on how similar the two intensities are. To better understand where this stimulus-induced difference originates from, we created TRFs of every 10-minute segment (30, 60, $70 \mathrm{~dB} A)$. Based on visual inspection of the topographies, we selected the centro-frontal channels as the channels of interest (Figure 7, black dots on panel A.).

Figure 7B shows 2 prominent peaks around 30-50 ms and 140-160 ms. Figure 7A shows the topographies of both peaks. To investigate the influence of stimulus intensity on the latency and amplitude of these peaks, we calculated the maximum for every participant per stimulus intensity between respectively 20 and $70 \mathrm{~ms}$, and 100 and $200 \mathrm{~ms}$ (= the peak value). The latency of the first peak is not significantly affected by stimulus intensity $(\mathrm{p}=0.55$, Skillings-Mack statistic $=1.20)$. The amplitude, on the other hand, is affected by stimulus intensity ( $\mathrm{p}=0.01$, Skillings-Mack statistic $=9.30$ ): When stimulus intensity increases from 30 to $60 \mathrm{~dB} A$, the amplitude of peak 1 increases $(\mathrm{p}=0.002, \alpha=0.025, \mathrm{CI}(95 \%)=[-0.010 ;-0.003], \mathrm{n}=20$, Wilcoxon signedrank test). However, when stimulus intensity further increases to $70 \mathrm{~dB} \mathrm{~A}$, peak 1 decreases as shown in Figure $7 \mathrm{~B}(\mathrm{p}=0.004, \alpha=0.025, \mathrm{CI}(95 \%)=[0.001 ; 0.007], \mathrm{n}=18$, Wilcoxon signed-rank test $)$. Peak 2 , in contrast to peak 1 , shows no significant effect of stimulus intensity on amplitude ( $p=0.212$, Skillings-Mack statistic $=3.10)$, but on peak latency $(p=0.036$, Skillings-Mack statistic $=6.65)$ : When stimulus intensity 
increases from 30 to $60 \mathrm{~dB}$ A the latency of peak 2 decreases $(\mathrm{p}=0.003, \alpha=0.025, \mathrm{CI}(95 \%)=[3.97 ; 23.81]$, $\mathrm{n}=20$, Wilcoxon signed-rank test). When stimulus intensity further increases to $70 \mathrm{~dB}$ A latency saturates $(\mathrm{p}=0.37, \alpha=0.025, \mathrm{CI}(95 \%)=[-23.81 ; 7.94], \mathrm{n}=18$, Wilcoxon signed-rank test $)$, as shown in Figure 7B

$$
\begin{array}{ccc} 
& \text { Peak } 1 & \text { Peak } 2 \\
10^{-3} & 30-50 \mathrm{~ms} & 140-160 \mathrm{~ms}
\end{array}
$$

A.
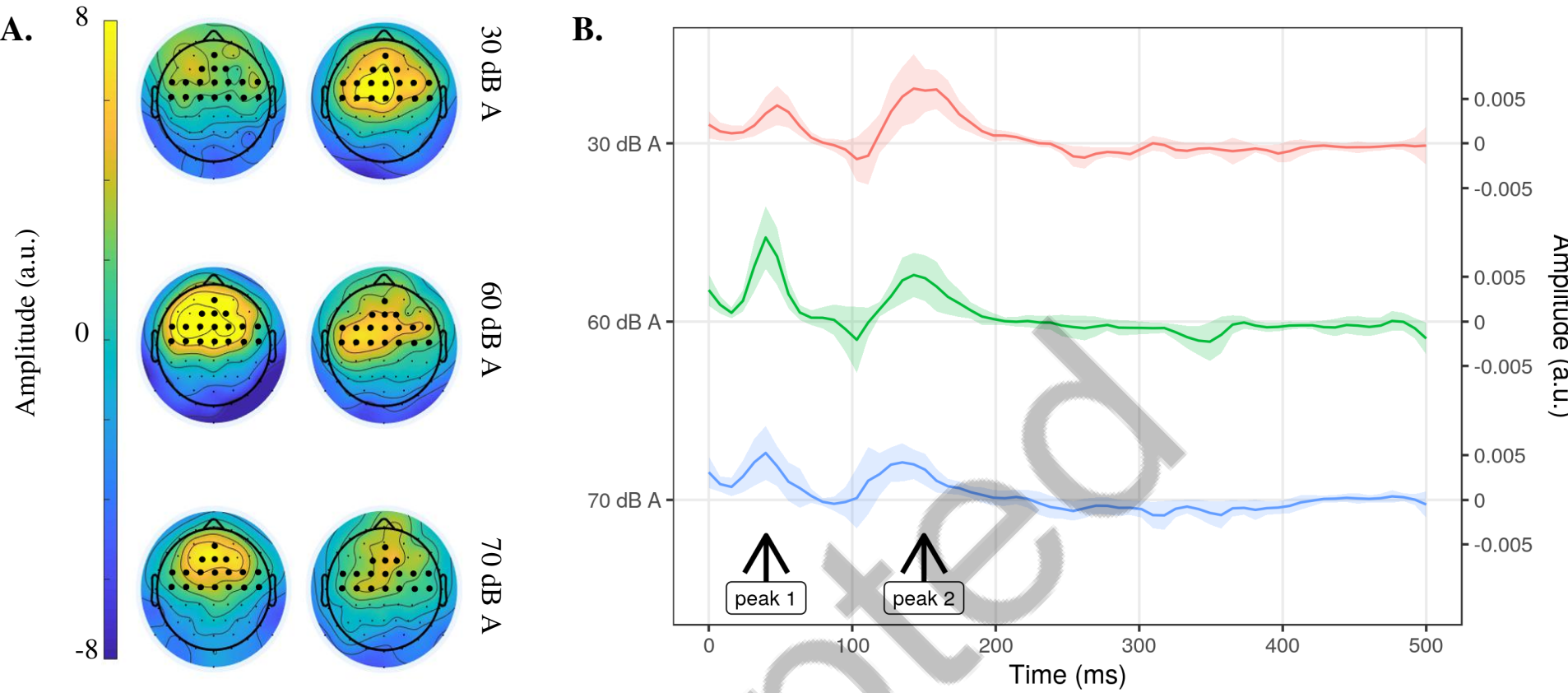

Figure 7. Temporal response functions (TRF) per stimulus intensity. A. Topographies showing mean TRF activity over subjects at the latencies of peak 1 and 2. Black dots represent the centro-frontal channel selection to create the TRFs in time-domain in panel B. B. Mean centro-frontal TRF over participants per intensity, shading is the standard error over participants.

\section{DISCUSSION}

We investigated whether stimulus intensity influences neural envelope tracking. We tested 20 normalhearing participants who listened to a story at different stimulus intensities. Neural envelope tracking is dependent on stimulus intensity in both TRF and envelope reconstruction analysis. However, provided that the decoder is applied on the same stimulus intensity as it was trained, envelope reconstruction is robust to stimulus intensity. This is promising for applications in hearing-impaired listeners requiring amplified speech. In addition, neural envelope tracking in the delta (but not theta) band seems to relate to speech intelligibility. 


\subsection{Effect of stimulus intensity on envelope reconstruction}

\subsubsection{Robust envelope reconstruction when training and testing on the same stimulus intensity}

We found no significant difference in neural envelope tracking between 30,60 , or $70 \mathrm{~dB}$ A when the decoder was applied on data of the same stimulus intensity as it was trained. This is consistent with the results reported by Ding and Simon (2012a) where no significant difference in neural envelope tracking was found when speech was attenuated by maximally $8 \mathrm{~dB}$ in a competing talker paradigm. Thus, we can tentatively conclude that presenting speech at different intensity levels, to, for example, ensure speech intelligibility in hearing-impaired listeners, does not influence the results. This implies that the enhancement of neural envelope tracking in hearing-impaired compared to normal-hearing listeners reported by Mirkovic et al. (2019) and Decruy et al. (2020b) is not driven by sound amplification. A plausible explanation for their enhanced results could be that hearing-impaired listeners need more effort to perform in the same way as normal-hearing listeners. Increased listening effort and increased attention has been associated with enhanced neural responses (Decruy et al., 2020a; Vanthornhout et al., 2019; Ding and Simon, 2012a b).

\subsubsection{Training and testing on different stimulus intensities affects neural envelope tracking}

Besides training and testing on equal stimulus intensities, as mentioned in Section 4.1.1, we also trained and tested on different stimulus intensities. When using different stimulus intensities to train and test the decoder on, reconstruction accuracy changes, especially in the theta band: a decoder trained on a low intensity enhances envelope reconstruction at low stimulus intensities, while a decoder trained on a high intensity enhances envelope reconstruction at high stimulus intensities. The more similar (ideally equal) the intensities for the training and testing part are, the better the reconstruction accuracy. Brain responses are dependent, both in amplitude and latency, on the intensity of the stimulus. This is shown in the TRF part of this study (section 3.3) and literature using non-speech stimuli (e.g., Hall, 2007; Picton et al., 2003; Van Eeckhoutte et al. 2016). When training a decoder on speech presented at a particular stimulus intensity, the decoder is optimized to decode brain responses with the latencies and amplitudes of that particular stimulus intensity. This underlines the importance of using a decoder stimulus similar to the testing stimulus for optimal tracking accuracy.

\subsection{Neural envelope tracking in the delta (but not theta) band relates to speech intelligibility}

Next, we compared neural envelope tracking results to speech intelligibility. We hypothesized that the better the acoustic speech envelope is encoded in the brain, the better the speech is understood (Ding and Simon, 2013; Di Liberto et al., 2018; Vanthornhout et al., 2018; Verschueren et al., 2020; Lesenfants et al., 2019; Decruy et al., 2019). In the delta band, stimulus intensity did not significantly influence neural envelope tracking between 20 (median SI $=80 \%$ ) and $80 \mathrm{~dB}$ A (median SI = 100\%). Only when including the lowest condition of $10 \mathrm{~dB}$ A (median $\mathrm{SI}=2.6 \%$ ) an increase of neural envelope tracking with increasing stimulus intensity could be found. This suggests that delta band tracking correlates with speech intelligibility rather than stimulus intensity. Only when speech intelligibility drops, neural envelope tracking in the delta band drops. On the other hand, in the theta band, an effect of stimulus intensity can be found even though speech intelligibility remains constant. This frequency band discrepancy between the delta and theta band could be explained by the hypothesis proposed by Ding and Simon (2013, 2014). They 
state that the delta band is related to the prosody of speech, while the theta band encodes syllabic-level acoustic features. Perhaps prosodic features are encoded more robustly in the brain because they are linked to top-down processing such as speech intelligibility, making them less sensitive to acoustic variations than theta band activity.

An additional argument for strengthening the hypothesis of speech intelligibility versus acoustics in respectively the delta and the theta band are the results in Figure 6 for the $20 \mathrm{~dB}$ A condition: Neural envelope tracking increases with increasing speech intelligibility in the delta band, but not in the theta band. This is in line with results reported by Molinaro and Lizarazu (2017). They also found that the theta band reflects perceptual processing of the auditory signal, while the delta band involves additional higher-order speech specific processing. In addition, studies by Meyer et al. (2017), Ding et al. (2014) and Vanthornhout et al. (2018) also show that especially delta band tracking correlates with respectively syntactic processing and speech intelligibility. Other evidence can be derived from the study of Ding et al. (2016): Only when speech was presented in a language that could be understood, neural responses occurred at the phrasal $( \pm 2 \mathrm{~Hz})$ and sentential rates $( \pm 1 \mathrm{~Hz})$ in addition to the acoustically present syllabic rate $( \pm 4 \mathrm{~Hz})$. These results show a hierarchy of neural processing timescales underlying speech intelligibility, which can be found within the delta band frequencies $(<4 \mathrm{~Hz})$. Moreover, these results showing that delta band tracking is relatively robust to stimulus intensity and could reflect speech intelligibility clarify the results reported by Verschueren et al. (2019): The increase in neural envelope tracking with increasing stimulus intensity in cochlear implant users could be related to speech intelligibility rather than stimulus intensity.

\subsection{Stimulus intensity affects temporal response functions}

In this study, we found two positive TRF peaks around 30-50 ms and 140-160 ms (Figure 7B). The different TRF peaks are thought to reflect different auditory processing stages varying from acoustic processing in the early latencies to more top-down driven processing (e.g., attention) in the later latencies. The first peak (30-50 ms) in this study could be related to the positive P1-like TRF peak occurring around $50 \mathrm{~ms}$ reported by, e.g., Ding and Simon (2012a, 2013); Petersen et al. (2017); Vanthornhout et al. (2019). The second peak, however, is more difficult to relate to literature. When measuring auditory evoked potentials, a large negative N1 peak occurs at a latency between 70-150 ms (Picton, 2011). In attention paradigms, N1-like TRF peaks have appeared to be important to detect selective attention (Ding and Simon, 2012a; Petersen et al., 2017). In this study, we found a prominent peak around the N1 latency (peak 2), but the polarity is inverted. It is important to note that we rereferenced our data to a common average of the channels to not bias topography patterns relative to the chosen channel. The disadvantage of common average rereferencing is mainly a more difficult interpretation of the absolute polarity (compared to only 1 reference) which makes it more difficult to relate our results to P1-N1 literature. As we are interested in how TRF magnitudes and latencies of different conditions relate to each other and not to an absolute number, we believe this is not a significant concern.

To investigate how the TRFs relate to stimulus intensity, we compared the amplitudes and latencies of peak 1 and 2 at different stimulus intensities. When stimulus intensity increased from 30 to $60 \mathrm{~dB} A$, the amplitude of peak $1(30-50 \mathrm{~ms})$ increased. This is in line with the observed increase of auditory evoked potential amplitudes as a function of stimulus intensity for non-speech stimuli (Moore and Rose, 1969, Picton et al., 1970; Adler and Adler, 1989; Billings et al., 2007; Hall, 2007; Picton et al., 2003; Van Eeckhoutte et al., 2016). However, when further increasing the stimulus intensity to $70 \mathrm{~dB}$ A, the amplitude of peak 1 decreased. A possible explanation for this non-linear behavior of TRF amplitude over stimulus intensity could be the saturation of the neural response. Several studies have reported amplitude saturation and decrease at around $70 \mathrm{~dB}$ (Butler et al., 1969; Picton et al., 1970; Adler and Adler, 1989), which is the same stimulus intensity as reported in this study.

In contrast to peak 1, the amplitude of peak 2 (140-160 ms) did not depend on stimulus intensity. This difference between peak 1 and 2 could be explained by the hypothesis that the early latencies of the TRF 
reflect acoustic processing of the speech (peak 1), while the later latencies (peak 2) of the TRF reflect higher-order speech processing (e.g., phonetic processing). An intensity-dependent peak 1 amplitude is expected, as a change in intensity is an acoustical change. A change in peak 2 amplitude, on the other hand, is not to be expected because a change in stimulus intensity between $30 \mathrm{~dB}$ A and $70 \mathrm{~dB}$ A does not influence speech understanding and thus higher-order speech processing. Ding and Simon (2013) reported similar results using speech in background noise. When more noise was added (the quality of the speech signal decreased), the amplitude of the TRF peak at $50 \mathrm{~ms}$ decreased ( peak 1), while the amplitude of the $100 \mathrm{~ms}$ peak ( peak 2) remained unchanged as long as speech could be understood.

Besides the amplitude, also the latency of the TRF peaks can be analyzed. The latency of peak 1 was not significantly affected by stimulus intensity. The latency of peak 2 , on the other hand, was affected by stimulus intensity: When stimulus intensity decreased, the latency of peak 2 increased from 30 to $60 \mathrm{~dB}$ A. We hypothesize that the TRF peak is delayed with lower stimulus intensities: although behaviorally measured speech intelligibility remains the same, the lower stimulus intensity makes it harder to understand the speech, resulting in a longer time needed to process the speech. This is similar to auditory evoked response potential literature using non-speech stimuli, where decreasing stimulus intensities result in increasing latencies (Hall, 2007; Picton et al., 2003; Van Eeckhoutte et al., 2016).

\subsection{Implications for applied research}

This study showed that neural envelope tracking depends on stimulus intensity for both the TRF and envelope reconstruction analysis. However, when the same stimulus intensity is used to train and test the decoder, envelope reconstruction seems not to be affected by stimulus intensity in contrast to TRFs. The apparent discrepancy between envelope reconstruction and TRFs is due to the underlying model: as the brain response function changes with intensity (as apparent from our TRF analysis), intensity-specific decoders are required. However, when this is the case, the brain response is similarly strong across intensities, as long as speech can be understood.

An interesting question that arises from this apparent discrepancy is which method (TRF vs. decoder) is optimal for measuring envelope tracking. We believe both methods have their advantages and disadvantages, depending on the intended purpose. TRFs can provide us with a lot more spatial and temporal information about the participants' speech processing. In addition, this method appears sensitive enough to find differences in acoustic properties of the participants' speech (e.g., stimulus intensity). The downside is that interpreting TRFs is time-consuming and can only be done by highly trained personnel. Decoders, on the other hand, are much more robust. Because of this robustness to, for example, stimulus intensity, decoders seem a reliable method when testing hearing-impaired participants with amplified speech and comparing them to normal-hearing peers. Also, decoders do not require any channel selection or peak-picking and return one correlation, making it easy to interpret.

When considering the application of envelope tracking as an objective measure of speech intelligibility, both methods could be complimentary. For example, decoders could be used to screen which patient has decreased speech intelligibility and should be referred to an audiologist. After referral, TRFs could, for example, be used to diagnose the specific speech intelligibility problem and decide on adequate treatment.

\subsection{Conclusion}

This study showed that neural envelope tracking depends on stimulus intensity in both TRF and envelope reconstruction analysis. However, provided that the decoder is applied to the same stimulus intensity as 
it was trained on, envelope reconstruction is robust to stimulus intensity. Therefore we can assume that intensity is not a confounder when testing hearing-impaired participants with amplified speech using the linear decoder approach. Second, we showed that higher envelope tracking could be obtained in the theta band when the intensity to train and test the decoder are similar. In addition, neural envelope tracking in the delta band appears to be correlated with speech intelligibility, showing the potential of neural envelope tracking as an objective measure of speech intelligibility. 
Acknowledgements: The authors would like to thank Lien Decruy, Sofie Keunen and Elise Verwaerde for their help in data acquisition.

Funding: This work was supported by the European Research Council (ERC) under the European Union's Horizon 2020 research and innovation programme [grant number 637424 (Tom Francart)]; the KU Leuven Special Research Fund [grant number OT/14/119]; and the Research Foundation Flanders (FWO) [grant numbers 1S10416N (Jonas Vanthornhout), 1S86118N (Eline Verschueren)].

\section{REFERENCES}

Adler, G., Adler, J., 1989. Influence of Stimulus Intensity on AEP Components in the 80- to 200Millisecond Latency Range. Audiology 28, 316-324.

Biesmans, W., Das, N., Francart, T., Bertrand, A., 2017. Auditory-inspired speech envelope extraction methods for improved EEG-based auditory attention detection in a cocktail party scenario. IEEE Transactions on Neural Systems and Rehabilitation Engineering 25, 402-412. doi:10.1109/TNSRE. 2016.2571900 .

Billings, C.J., Tremblay, L., Souza, P.E., Binns, A., 2007. Effects of Hearing Aid Amplification and Stimulus Intensity on Cortical Auditory Evoked Potentials. Audiol Neurotol 12, 234-246. doi:10. $1159 / 000101331$.

Brodbeck, C., 2017. Eelbrain: 0.25. Zenodo. doi:10.5281/zenodo.1186450.

Butler, R.A., Keidel, W.D., Spreng, M., 1969. An Investigation of the Human Cortical Evoked Potential Under Conditions Of Monaural and Binaural Stimulation. Acta Oto- Laryngologica 68, 317-326. doi:10.3109/00016486909121570

Crosse, M.J., Liberto, G.M.D., Bednar, A., Lalor, E.C., 2016. The Multivariate Temporal Response Function ( mTRF) Toolbox : A MATLAB Toolbox for Relating Neural Signals to Continuous Stimuli. Frontiers in Human Neuroscience 10, 604. doi:10.3389/fnhum. 2016.00604.

Das, N., Biesmans, W., Bertrand, A., Francart, T., 2016. The effect of head-related filtering and earspecific decoding bias on auditory attention. Journal for neural engineering 13, 056014. doi:10.1088/ 1741-2560/13/5/056014.

David, S.V., Mesgarani, N., Shamma, S.A., 2007. Estimating sparse spectro-temporal receptive fields with natural stimuli. Network: Computation in Neural Systems 18, 191-212. doi:10.1080/ 09548980701609235.

Decruy, L., Lesenfants, D., Vanthornhout, J., Francart, T., 2020a. Top-down modulation of neural envelope tracking : The interplay with behavioral, self-report and neural measures of listening effort. European Journal of Neuroscience 00, 1-19. doi:10.1111/ejn.14753.

Decruy, L., Vanthornhout, J., Francart, T., 2019. Evidence for enhanced neural tracking of the speech envelope underlying age- related speech-in-noise difficulties. Journal of neurophysiology 122, 601-615. doi:10.1152/jn.00687.2018.

Decruy, L., Vanthornhout, J., Francart, T., 2020b. Hearing impairment is associated with enhanced neural tracking of the speech envelope. Hearing Research 393, 107961. doi:DOI : j. heares. 2020. 107961 .

Di Liberto, G.M., Lalor, E.C., Millman, R.E., 2018. Causal cortical dynamics of a predictive enhancement of speech intelligibility. NeuroImage 166, 247-258. doi:10.1016/j.neuroimage.2017.10. 066 .

Ding, N., Chatterjee, M., Simon, J.Z., 2014. Robust cortical entrainment to the speech envelope relies on the spectro-temporal fine structure. NeuroImage 88, 41-46. URL: http://dx.doi.org/ $10.1016 / j$.neuroimage.2013.10.054, doi:10.1016/j.neuroimage.2013.10.054, arXiv:NIHMS150003.

Ding, N., Melloni, L., Zhang, H., Tian, X., Poeppel, D., 2016. Cortical tracking of hierarchical linguistic structures in connected speech. Nature Neuroscience 19, 158-64. URL: http://www.ncbi.nlm. nih.gov/pubmed/26642090, doi:10.1038/nn.4186, arXiv:15334406.

Ding, N., Simon, J.Z., 2012a. Emergence of neural encoding of auditory objects while listening to competing speakers. Proceedings of the National Academy of Sciences of the United States of America 109, 11854-9. doi:10.1073/pnas.1205381109.

Ding, N., Simon, J.Z., 2012b. Neural coding of continuous speech in auditory cortex during monaural and dichotic listening. Journal of Neurophysiology 107, 78-89. doi:10.1152/ jn.00297.2011. 
Ding, N., Simon, J.Z., 2013. Adaptive Temporal Encoding Leads to a Background-Insensitive Cortical Representation of Speech. Journal of Neuroscience 33, 5728-5735. doi:10.1523/ JNEUROSCI. 5297-12.2013.

Ding, N., Simon, J.Z., 2014. Cortical entrainment to continuous speech: functional roles and interpretations. Frontiers in Human Neuroscience 8,1-7. doi:10.3389/fnhum.2014.00311.

Francart, T., van Wieringen, A., Wouters, J., 2008. APEX 3: a multi-purpose test platform for auditory psychophysical experiments. Journal of Neuroscience Methods 172, 283-293.

Hall, J.W., 2007. New handbook of auditory evoked responses. Allyn \& Bacon, Boston.

Haufe, S., Meinecke, F., Görgen, K., Dähne, S., Haynes, J.d., Blankertz, B., Bießmann, F., 2014. NeuroImage On the interpretation of weight vectors of linear models in multivariate neuroimaging. NeuroImage 87, 96-110. doi:10.1016/j.neuroimage.2013.10.067.

Iotzov, I., Parra, L., 2019. EEG can predict speech intelligibility To. J. Neural Eng. 16, 036008 (11p). doi:10.1088/1741-2552/ab07fe.

Lalor, E.C., Pearlmutter, B.A., Reilly, R.B., McDarby, G., Foxe, J.J., 2006. The VESPA: A method for the rapid estimation of a visual evoked potential. NeuroImage 32,1549-1561. doi:10.1016/j neuroimage.2006.05.054.

Lesenfants, D., Francart, T., 2020. The interplay of top-down focal attention and the cortical tracking of speech. Scientific Reports 10, 6922. doi:10.1038/s41598-020-63587-3.

Lesenfants, D., Vanthornhout, J., Verschueren, E., Decruy, L., Francart, T., 2019. Predicting individual speech intelligibility from the neural tracking of acoustic- and phonetic-level speech representations. Hearing Research 380,1-9. doi:10.1016/j.heares.2019.05.006.

Meyer, L., Henry, M.J., Gaston, P., Schmuck, N., Friederici, A.D., 2017. Linguistic Bias Modulates Interpretation of Speech via Neural Delta-Band Oscillations. Cerebral Cortex 27, 4293-4302. doi:10. $1093 /$ cercor/bhw228.

Mirkovic, B., Debener, S., Schmidt, J., Jaeger, M., Neher, T., 2019. Effects of directional sound processing and listener' s motivation on EEG responses to continuous noisy speech : Do normal-hearing and aided hearing-impaired listeners differ ? Hearing Research 377, 260-270. doi:10.1016/j. heares .2019. 04.005 .

Molinaro, N., Lizarazu, M., 2017. Delta(but not theta)-band cortical entrainment involves speechspecific processing. European Journal of Neuroscience 9, 1-9. doi:10.1111/ejn.13811, arXiv:0608246v3.

Moore, E.J., Rose, D.E., 1969. Variability of Latency and Amplitude of Acoustically Evoked Responses to Pure Tones of Moderate to High Intensity. International audiology 8, 172-181. doi:10.3109/ 05384916909070204 .

O’Sullivan, J.A., Power, A.J., Mesgarani, N., Rajaram, S., Foxe, J.J., Shinn-Cunningham, B.G., Slaney, M., Shamma, S.A., Lalor, E.C., 2015. Attentional Selection in a Cocktail Party Environment Can Be Decoded from Single-Trial EEG. Cerebral Cortex 25, 1697-1706. doi:10.1093/ cercor/bht 355.

Petersen, E.B., Wöstmann, M., Obleser, J., Lunner, T., 2017. Neural tracking of attended versus ignored speech is differentially affected by hearing loss. Journal of Neurophysiology 117, 18-27. doi:10.1152/jn.00527.2016.

Picton, T.W., 2011. Human Auditory Evoked Potentials. Plural Publishing inc., San Diego.

Picton, T.W., Goodman, W.S., Bryce, D.P., 1970. Amplitude of Evoked Responses to Tones of High Intensity. Acta Oto-Laryngologica, 70, 77-82. doi:10.3109/00016487009181862.

Picton, T.W., John, M.S., Dimitrijevic, A., Purcell, D., 2003. Human auditory steady-state responses. internation Journal of Audiology 42, 177-219. doi:10.3109/14992020309101316.

Presacco, A., Simon, J.Z., Anderson, S., 2019. Speech-in-noise representation in the aging midbrain and cortex : Effects of hearing loss. PloS one , 1-26doi:https://doi.org/10.1371/journal. pone.0213899March.

Shannon, R.V., Zeng, F.G., Kamath, V., Wygonski, J., Ekelid, M., 1995. Speech recognition with primarily temporal cues. Science 270, 303-304.

Skillings, J.H., Mack, G.A., Mack, G.A., 1981. On the Use of a Friedman-Type Statistic in Balanced and Unbalanced Block Designs 23, 171-177.

Somers, B., Francart, T., Bertrand, A., 2018. A generic EEG artifact removal algorithm based on the multi-channel Wiener filter. Journal of neural engineering 15. doi:10.1088/1741-2552/aaac92.

Van Eeckhoutte, M., Wouters, J., Francart, T., 2016. Auditory steady-state responses as neural correlates of loudness growth. Hearing Research 342, 58-68. doi:10.1016/j.heares.2016.09.009. 
